\title{
Polarimetric observations of the comet Hale-Bopp
}

\author{
S. Ganesh, U.C. Joshi, K.S. Baliyan, and M.R. Deshpande \\ Physical Research Laboratory, Ahmedabad - 380 009, India \\ Received August 21; accepted October 20, 1997
}

\begin{abstract}
The continuum polarization observations, spread over a period from October 1996 to May 1997, of the coma region of comet Hale Bopp are made at various phase angles, varying from 17.1 to 47.4 degrees, using IAU/IHW filter set. The linear polarization measured through 3650, 4845 and $6840 \AA$ bands shows stronger wavelength dependence as compared to what is seen in comet Halley at similar phase angles. Comparisons are made with Comet Halley polarization properties and it is inferred that Comet Hale Bopp has smaller grains than those found for Comet Halley. However, this comet shows similarity with Comet Halley so far as the albedo of the grains is concerned. This comet appears to belong to the high polarization class in the classification scheme based on polarization characteristics.
\end{abstract}

Key words: polarization — comet: P/Halley; Hale-Bopp C/1995 O1

\section{Introduction}

The continuum radiation in comets is polarized due to scattering of sunlight by the dust particles present in the comet's atmosphere. The degree of polarization and its direction depend upon the size distribution, composition of particles (which can be defined in terms of an average complex refractive index), scattering angle and wavelength of the incident solar radiation. A study of the polarization of continuum radiation in comets is therefore a powerful means to determine the characteristics of the cometary dust grains (Ohman 1941; Blackwell \& Willstrop 1957; Bappu et al. 1987; Sen et al. 1991a,b). During Halley's 1986 apparition (and subsequently for more recent comets) a large number of observations of cometary continuum polarization were obtained by several groups (see Joshi et al. 1997).

Phase curves of various comets appear to be similar (Dollfus et al. 1988; Levasseur-Regourd et al. 1996, and references therein). Such phase curves seem to be typical of fluffy regoliths, or irregular dust particles with sizes greater than $1 \mu \mathrm{m}$ (Giese et al. 1978) and are similar to those expected from aggregates of interstellar dust (Greenberg \& Gustafson 1981). The nature of the phase curves is expected to provide clues to the physical properties of the dust.

Levasseur-Regourd et al. (1996) have classified the comets in two classes on the basis of polarimetric properties. These two classes of comets are distinct only for the phase angles above $40^{\circ}$. Therefore, it is important to have polarimetric data covering a wide range of phase angles, especially above $40^{\circ}$ to classify the comet in either category. Further observations of comets over a large range of phase angles and continuum wavelengths is necessary before making a definitive statement about the difference in the properties of cometary dust for the two classes.

Comet Hale-Bopp C/1995 O1 is an intrinsically bright comet and having been discovered two years before perihelion, it has provided sufficient time to the astronomers to plan their observations. Furthermore, it was possible to make observations over a large range of phase angles for this comet although the largest phase observed was $47^{\circ}$.

In this article we report continuum linear polarization observations made during the period from October 1996 to May 1997. On the basis of this data we discuss the nature of dust grains and compare Hale-Bopp characteristics with some other comets.

\section{Observations}

Observations of Comet Hale-Bopp C/1995 O1 were taken with the optical polarimeter (Deshpande et al. 1985; Joshi et al. 1987) mounted at the Cassegrain focus of the $1.2 \mathrm{~m}$ telescope at Mt. Abu Infrared Observatory of Physical Research Laboratory, India.

The optical polarimeter is equipped with the IAU/IHW filter set. These filters were acquired about a decade ago and have been kept with care in a dry atmosphere to preserve them. However, to be sure of their transmission characteristics, we determined the transmission curves of these filters before the start of present observations. We found that (with the exception of the $\mathrm{CO}^{+}(4260 \AA)$ filter curve) they compare very well with the transmission characteristics supplied by the 
manufacturers without any significant deterioration. The polarization in the three continuum bands $(3650 / 80$, $4845 / 65,6840 / 90$, all figures in $\AA$ ) is reported here. It is now known that these continuum filters suffer from the contamination from a previously underestimated cometary molecular emission (Schleicher 1996). However, the degree of polarization and position angle are not expected to be affected significantly by small contamination. Also, the observations with these filters facilitate comparison with comets observed earlier with same set of filters, hence their continued use is justified.

The observations were conducted during the period from October 1996 to May, 1997. The apertures employed for these observations were of size 52.4 and 26.5 arcsec. The observations were made alternately on the photocenter of the comet and the region of sky nearly 45 arcmin away, in the anti-tailward direction. This procedure was used to take care of the sky polarization. Polarization standard stars, HD 14433, 9 Gem, o Sco (Serkowski 1973), were used to calibrate the observed position angle. A zero polarization star, HD 202575, was used to determine the instrumental polarization which was found to be negligible $(\leq 0.03 \%)$ compared to the errors in the observation. The instrument's performance was tested for $100 \%$ linear polarization using a Glan prism and it was found to be $98.7 \%$ efficient. The details of the data reduction procedures are given by Joshi et al. (1987).

\section{Discussion}

Table 1 lists the observed values of polarization in the three continuum filters along with the apertures used and the phases at which the observations were carried out. The heliocentric and geocentric distances at the time of observation are also given. There are six observing runs before perihelion passage (1st April 1997) and seven during the post-perihelion period.

The observations are mostly made with an aperture of 26.5 arcsec diameter. However the Earth-Comet distance being different for different observations, sampled area of the comet is different. This might cause some change in the degree of polarization. The inner coma region of comets has been found to be quite heterogeneous in spatial distribution of polarization by various researchers (Renard et al. 1996). In the case of Halley phase curves for the inner coma (radius $\leq 5000 \mathrm{~km}$ ) and the outer region (radius $\geq 10000 \mathrm{~km}$ ) appear different (Dollfus et al. 1988). This maybe due to either a difference in the microtexture of the dust grains (Dollfus 1989) or a difference in dust size distribution. But in the case of Halley, a fair degree of agreement was noticed among all the observers, as long as the polarization was estimated over the whole coma with a large aperture (which averages out the effect of the heterogeneity of the inner coma) aimed at the photometric center. In all our observations the linear scale sampled on the comet is greater than $\approx 13000 \mathrm{~km}$ radius which is much larger than the inner coma region. In order to check the polarization behaviour with respect to the area sampled on the comet, we observed the comet through two different apertures on 1st March 1997 (cf. Table 1). We note that within the errors of observation there is no significant difference for the two apertures. The linear scales corresponding to apertures of 26.5 and 52.4 arcsec are respectively 14318 and $28313 \mathrm{~km}$ radius. This means that the comparison of polarization observations on different dates is meaningful as long as the sampled linear size is in the range 14000 to $28000 \mathrm{~km}$ radius. On the other hand, during the October, November, 1996 observations the area sampled is much larger (linear scale $>55000 \mathrm{~km}$ radius) and light from the tail region also contributes to the observed polarization. During these (October, November, '96) observations, the phase angles are between $17^{\circ}-19^{\circ}$ and the observed polarization is found to have the position angle parallel to the plane of scattering i.e. the polarization is negative. The comet being fainter during this period, the observed errors in polarization measurements are large, especially in the $u$-band $(3650 \AA$ ) observations. In the other two continuum bands, the $\mathrm{S} / \mathrm{N}$ ratio is better than three and therefore the numbers are significant. The polarization values measured on November 1 and November 2, 1996 differ significantly though the phase angles and comet distances from Sun and Earth are nearly same. This could be due to different levels of activity on the above two dates.

In Fig. 1, we have shown the observed polarization for all the three wavelengths as a function of the phase at the time of observation. The solid line is a theoretical curve adopted from Sen et al. (1991b) for refractive index and dust distribution estimated for Halley's comet. It is clear from the figure that there is a significant deviation between the observed polarization values of Comet HaleBopp and the phase curves for Halley, especially at the larger phase angles. On the basis of their study of observations of various comets, Levasseur-Regourd et al. (1996) have suggested the existence of two classes of comets: low polarization and high polarization classes. Comparing our observations with Figs. 3a and 3b of their paper, it is evident that at phase angles above $30^{\circ}$ the polarization observed in Comet Hale-Bopp is the highest observed till now. If the classification suggested by Levasseur-Regourd et al. (1996), is applied, the comet Hale-Bopp will belong to high polarization class of comets.

A polynomial fit to the observed data (with the boundary conditions of zero polarization at $0^{\circ}$ and $180^{\circ}$ phase angles) gives the values of the inversion angle and the slope at inversion and these are listed in Table 2 for the three continuum wave bands. The values of the slope at inversion, $h$, for the $3650 \AA$ and $4845 \AA$ wavelengths obtained for this comet are close to those for Halley $(\approx 5000 \AA)$ while for the $6840 \AA$ wavelength the slope for this comet is clearly larger. The parameter $h$ is a direct indicator of the albedo (see Fig. 1 in Dollfus 1989). The albedo of the dust grains in this comet is similar to that of Halley for 
Table 1. Polarization observations of Comet Hale-Bopp C/1995 O1 obtained using continuum filters

\begin{tabular}{|c|c|c|c|c|c|c|c|c|}
\hline UT date & $r$ & $\delta$ & phase & Aperture & Wavelength & & lariza & \\
\hline & $\mathrm{AU}$ & $\mathrm{AU}$ & deg & $\operatorname{arcsec}($ dia.) & $\AA$ & $\% P$ & $E_{\mathrm{p}}$ & $\theta$ \\
\hline 1996 October 16.64 & 2.70 & 3.04 & 18.8 & 52.4 & 3650 & -3.11 & 2.40 & 78 \\
\hline & & & & 52.4 & 4845 & -3.13 & 0.85 & 105 \\
\hline & & & & 52.4 & 6840 & -3.85 & 1.16 & 102 \\
\hline 1996 November 1.60 & 2.52 & 3.05 & 17.2 & 52.4 & 3650 & -2.69 & 2.17 & 119 \\
\hline & & & & 52.4 & 4845 & -1.26 & 0.27 & 70 \\
\hline & & & & 52.4 & 6840 & -2.01 & 0.22 & 81 \\
\hline 1996 November 2.6 & 2.50 & 3.05 & 17.1 & 52.4 & 4845 & -0.55 & 0.37 & 48 \\
\hline & & & & 52.4 & 6840 & -1.12 & 0.27 & 68 \\
\hline 1997 February 13.02 & 1.23 & 1.77 & 32.6 & 26.5 & 3650 & 3.94 & 0.25 & 60 \\
\hline & & & & 26.5 & 4845 & 4.40 & 0.07 & 53 \\
\hline & & & & 26.5 & 6840 & 5.67 & 0.08 & 53 \\
\hline 1997 February 28.02 & 1.08 & 1.50 & 41.1 & 26.5 & 3650 & 7.87 & 0.11 & 61 \\
\hline & & & & 26.5 & 4845 & 9.33 & 0.04 & 62 \\
\hline & & & & 26.5 & 6840 & 12.16 & 0.04 & 63 \\
\hline 1997 March 1.01 & 1.07 & 1.49 & 41.7 & 26.5 & 3650 & 7.83 & 0.28 & 58 \\
\hline & & & & 26.5 & 4845 & 9.43 & 0.07 & 59 \\
\hline & & & & 26.5 & 6840 & 11.63 & 0.05 & 59 \\
\hline & & & & 52.4 & 3650 & 8.08 & 0.10 & 60 \\
\hline & & & & 52.4 & 4845 & 9.57 & 0.05 & 60 \\
\hline & & & & 52.4 & 6840 & 11.73 & 0.07 & 60 \\
\hline 1997 April 2.59 & 0.91 & 1.36 & 47.0 & 26.5 & 3650 & 10.82 & 0.30 & 120 \\
\hline & & & & 26.5 & 4845 & 12.20 & 0.06 & 116 \\
\hline & & & & 26.5 & 6840 & 14.63 & 0.04 & 110 \\
\hline 1997 April 3.62 & 0.92 & 1.37 & 46.6 & 26.5 & 3650 & 10.84 & 0.50 & 121 \\
\hline & & & & 26.5 & 4845 & 12.22 & 0.03 & 120 \\
\hline & & & & 26.5 & 6840 & 14.56 & 0.10 & 119 \\
\hline 1997 April 4.59 & 0.92 & 1.38 & 46.2 & 26.5 & 3650 & 10.38 & 0.20 & 127 \\
\hline & & & & 26.5 & 4845 & 11.86 & 0.05 & 121 \\
\hline & & & & 26.5 & 6840 & 14.15 & 0.06 & 121 \\
\hline 1997 April 10.61 & 0.93 & 1.44 & 43.1 & 26.5 & 3650 & 8.82 & 0.13 & 133 \\
\hline & & & & 26.5 & 4845 & 10.24 & 0.04 & 132 \\
\hline & & & & 26.5 & 6840 & 12.27 & 0.06 & 131 \\
\hline 1997 April 28.6 & 1.03 & 1.72 & 31.4 & 26.5 & 3650 & 4.50 & 0.25 & 165 \\
\hline & & & & 26.5 & 4845 & 4.50 & 0.05 & 168 \\
\hline & & & & 26.5 & 6840 & 5.67 & 0.07 & 161 \\
\hline 1997 April 29.6 & 1.04 & 1.74 & 30.8 & 26.5 & 3650 & 3.15 & 0.40 & 167 \\
\hline & & & & 26.5 & 4845 & 3.98 & 0.09 & 164 \\
\hline & & & & 26.5 & 6840 & 5.05 & 0.09 & 166 \\
\hline 1997 May 4.6 & 1.08 & 1.83 & 27.7 & 26.5 & 3650 & 2.30 & 1.00 & 144 \\
\hline & & & & 26.5 & 4845 & 2.73 & 0.10 & 165 \\
\hline & & & & 26.5 & 6840 & 3.75 & 0.10 & 160 \\
\hline
\end{tabular}

the shorter wavelengths. The albedo is very low i.e. the comet grains are dark. The total brightness of the comet Hale-Bopp was far below the expected brightness - the presence of darker dust grains may be one of the reasons. The inversion angle is related to the fluffiness of the dust grains - fluffier the particle, larger the phase angle at which inversion occurs. In the present case the inversion angle is found to be dependent on the wavelength. This may be due to the fluffy nature of the grains.

Figure 2 displays the wavelength dependence of polarization at different phase angles. Shown with a dotted line is the wavelength dependence of Comet Halley (Kikuchi et al. 1987) for one phase angle. This figure shows that continuum polarization in Comet Hale-Bopp has a very strong wavelength dependence. The degree of polarization
Table 2. Polarization properties obtained from polynomial fit to the observed data(Table 1)

\begin{tabular}{cccc}
\hline $\begin{array}{c}\text { Wavelength } \\
\text { in } \AA\end{array}$ & $\begin{array}{c}P_{\min } \\
\text { @(phase })\end{array}$ & $\begin{array}{c}\text { Inversion angle Slope at inversion } \\
V_{0}\end{array}$ \\
\hline 3650 & $-2.1 \%\left(@ 10^{\circ}\right)$ & $23.6^{\circ}$ & $0.29 \% /^{\circ}$ \\
4845 & $-1.8 \%\left(@ 10^{\circ}\right)$ & $21.7^{\circ}$ & $0.28 \% /^{\circ}$ \\
6840 & $-2.2 \%\left(@ 9^{\circ}\right)$ & $21.5^{\circ}$ & $0.35 \% /^{\circ}$ \\
\hline
\end{tabular}

increases with increasing wavelength which is a marked difference from Comet Halley whose wavelength dependence is more flatter. This wavelength dependence is found to increase with increasing phase angles. This is expected for smaller (compared to Halley) sized dust grains. 

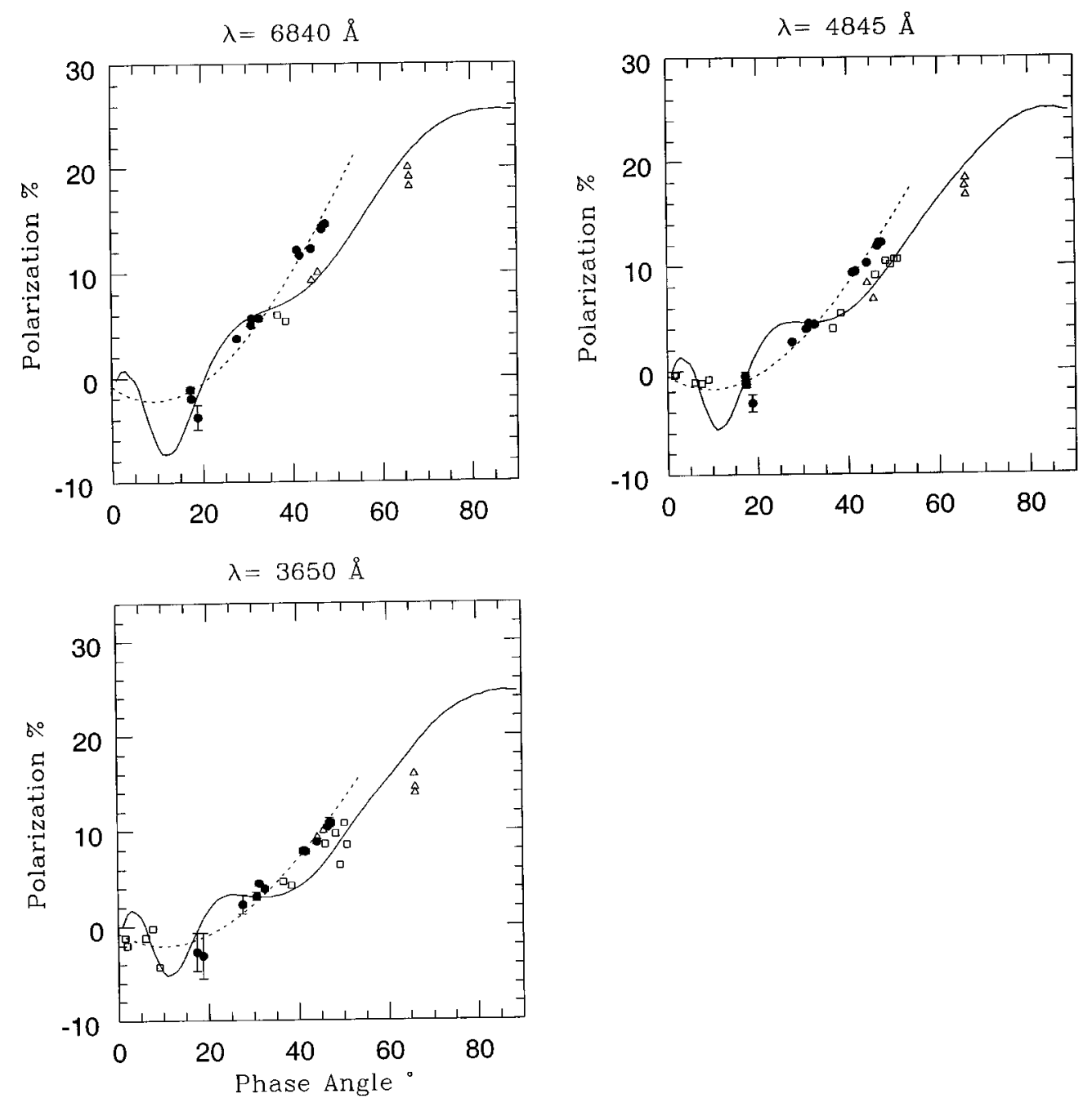

Fig. 1. The observed percentage polarization as a function of phase angle for the three continuum bands. Filled circles represent the data for Comet Hale-Bopp, open triangles and open squares are for comet Halley, taken from two sources: Sen et al. (1991b) and Kikuchi et al. (1987) respectively. The solid line is the best fit model curve for Halley data (Sen et al. 1991b). Dotted curve is a polynomial fit to the present data

\section{Conclusion}

We have measured the continuum band polarization in Comet Hale-Bopp C/1995 O1. The polarization near perihelion is largest in comet Hale Bopp than observed so far in any comet at the corresponding phase angles. In the classification scheme based on the polarization (Levasseur-Regourd et al. 1996) this comet belongs to the high polarization class of comets. Comet Hale-Bopp exhibits stronger wavelength dependence of polarization than other comets. We conclude that Comet Hale-Bopp $\mathrm{C} / 1995$ O1 contains smaller grains compared to comets Halley or Austin. The albedo of the dust particles in this comet is found to be very low, a case similar to Comet Halley.

Acknowledgements. The authors would like to thank Prof. K.S. Krishnaswamy for useful discussions; Donald Yeomans for providing the ephemeris; and engineers N.M. Vadher, A.B. Shah and V.M. Shah for their help during observations. Use of the NASA ADS Abstract Service is acknowledged. This work is funded by Department of Space, Government of India.

\section{References}

Bappu M.V.K., Sivaraman K.R., Bhatnagar A., Natrajan V., 1967, MNRAS 136, 19

Blackwell D.E., Willstrop R.V., 1957, MNRAS 117, 590

Deshpande M.R., Joshi U.C., Kulshrestha A.K., Bansidhar, et al., 1985, Bull. Astron. Soc. India 13, 157

Dollfus A., Bastien P., Le Borgne J.F., Levasseur-Regourd A.C., Mukai T., 1988, A\&A 206, 348

Dollfus A., 1989, A\&A 213, 469

Giese R.H., Weiss K., Zerull R.H., Ono T., 1978, A\&A 65, 265

Greenberg J.M., Gustafson B.A.S., 1981, A\&A 93, 35

Joshi U.C., Deshpande M.R., Sen A.K., Kulshrestha A.K., 1987, A\&A 181, 31 


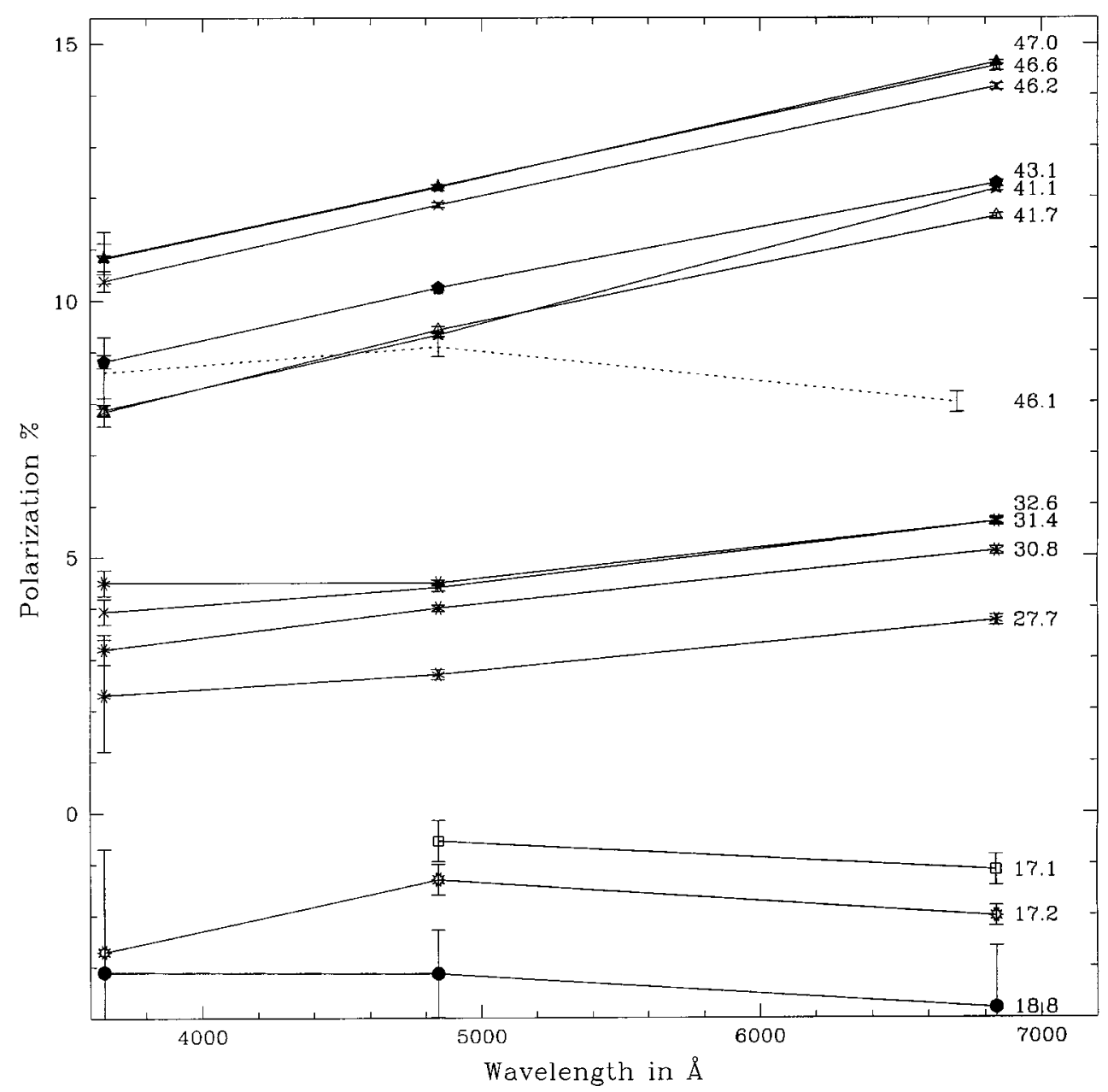

Fig. 2. Wavelength dependence of polarization for Comet Hale-Bopp, as observed at different phases; Phase angles are mentioned adjacent to the respective curves. Comet Halley data (Kikuchi et al. 1987) for one phase (46.1) close to observations of Hale-Bopp at phase 46.2 is also plotted in the figure (dotted line)

Joshi U.C., et al., 1997, A\&A 319, 694

Kikuchi S., Mikami Y., Mukai T., Mukai S., Hough T., 1987, A\&A 187, 689

Levasseur-Regourd A.-C., et al., 1996, A\&A 313, 327

Ohman Y., 1941, Stockholm Obs. Ann. 13, 11

Renard J.B., Hadamcik H., Levasseur-Regourd A.-C., 1996, A\&A 316, 263

Schleicher D., 1996 (private communication)
Sen A.K., Joshi U.C., Deshpande M.R., 1991a, MNRAS 253, 738

Sen A.K., Deshpande M.R., Joshi U.C., Rao N.K., Raveendran A.V., 1991b, A\&A 242, 496

Serkowski K., 1974, in Planets, stars and nebulae studied through photopolarimetry, Gehrels T. (ed.). Univ. of Arizona Press 